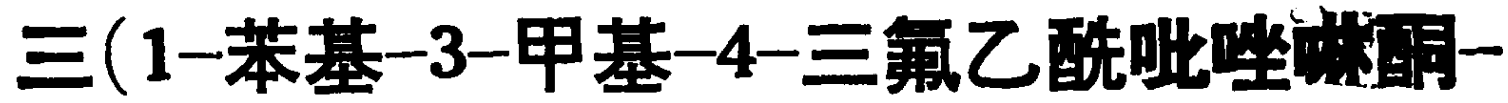 5 )二(三苯基氧化膦)合钕（III） 协萃络合物的合成和晶体结构*
}

\author{
黄春辉 邓安平 徐光宪 \\ (北京大学化学系) \\ 䇣方明刘小兰 \\ (天津师范大学化学系)
}

\section{关链词 1-苯基-3-甲基-4-三氧乙酰基吡叫啉酮-5、三苯基算化膦钽、晶体结构}

自从 Jensen 于 1959 年合成了一系列 1-苯基-3-甲基-4-酰基吡唑啉酠-5 以后 ${ }^{[3]}$, 作为 一类重要的萃取剂和位移试剂, 它们已被广泛地研究和应用 ${ }^{[3]}$. 我们曾合成了一系列在 4-位 上为卤代乙酰基的吡唑啉醖, 并就它对稀土元素的萃取性能进行了研究 ${ }^{[3]}$. 这类萃取剂与中 生萃取剂一起具有一定的协萃性能 ${ }^{[4]}$. 作为系统研究的一部分, 我们合成了稀土系列的 1-苯 基-3-甲基-4-三氟乙酰基吡唑啉酠-5（HPMTFP）和三苯基氧化膦（Ph, PO）的协萃络合物 $\mathrm{RE}(\mathrm{PMTFP})_{3} \cdot\left(\mathrm{Ph}_{3} \mathrm{PO}\right)_{2}\left(\mathrm{RE}=\mathrm{La}-\mathrm{Lu}\right.$ 及 $\mathrm{Y}$, 除 $\mathrm{Pm}$ 外), 并对其中 $\mathrm{Nd}(\mathrm{PMTFP})_{3}\left(\mathrm{Ph}_{3} \mathrm{PO}\right)_{2}$ 的红外光谱及单晶结构进行分析研究.

\section{一、合 成 实 验}

1. 试制 HPMTFP: 按文献 [1]方法合成。酮式产物熔点: $144-145^{\circ} \mathrm{C}$. $\mathrm{Ph}_{3} \mathrm{PO}$ ：德国 MERCK-Schuchardt 公司生产, 纯度 $98 \%$. 熔点: $154-157{ }^{\circ} \mathrm{C}$. 稀土纯度 $99.9 \%$,其余试剂均为分析纯.

2. 络合物晶体的合成将 $3 \mathrm{mmol}$ HPMTFP, $2 \mathrm{mmol} \mathrm{Ph}_{3} \mathrm{PO}$ 与 $1 \mathrm{mmol} \mathrm{Nd}\left(\mathrm{NO}_{3}\right)_{3}$ 溶 于 $25 \mathrm{ml}$ 无水乙醇中, 加入 $\mathrm{NaOH}$ 溶液调节 $\mathrm{pH}$ 至 3-4, 放置, 有紫红色晶体生成, 取出 用无水乙醇洗涤后在空气中干燥. 元素分析结果百分值为: C 57.40 (实验值 57.33)，N 5.56 (5.57), H 3.89(3.61).

$\mathrm{Nd}(\mathrm{PMTFP}):\left(\mathrm{Ph}_{3} \mathrm{P}=\mathrm{O}\right)_{2}$ 的红外光谱: 在 Nicolet-7199B 型 FTIR 光谱仪上采用溴 化钾压片在中红外区 $\left(400-4000 \mathrm{~cm}^{-1}\right)$ 测定了络合物的红外光谱.

在自由的 $\mathrm{Ph}_{3} \mathrm{PO}$ 中 $\mathrm{P}=\mathrm{O}$ 键的红外吸收峰位于 $1190 \mathrm{~cm}^{-1}$, 配位后红移 至 $1154 \mathrm{~cm}^{-1}$, $\Delta \gamma-36 \mathrm{~cm}^{-1}$, 这是由于氧与钕的配位, 使 $\mathrm{P}=0$ 键有一定程度的削弱听致.

本文 1988 年 3 月 26 日收到. 1988 年 8 月 20 日收到修改稿.

- 国家自然科学基金资助项目. 
在 $\beta$-二阔分子中,由于存在着烯醇式和酮式的互变异构平衡，因此自由的 HPMTFP 㟧 红外吸收光谱在 $3429 \mathrm{~cm}^{-1}$ 附近有一个较强的宽带吸收。这是醇式 HPMTFP 中内氞键的 吸收，而整合物的红外光谱在 $3413 \mathrm{~cm}^{-1}$ 附近有一个强度很弱的不明显的宽带吸收, 说明 p二阙上的两个氧参加配位后分子中内氢链不复存在. 在比较了 HPMTFP 和它的络合物的 红外光谱后. 我们发现在 $\beta$-二酮中 $\mathrm{C}=0$ 键的两个吸收峰在配位后一个发生红移, 一个 发生紫移的现象, 这一现象在我们所研究的这一系列络合物中都有表现, 自由的 HPMTFP 配体中其 $C=0$ 键的吸收峰出现在 1682 和 $1625 \mathrm{~cm}^{-1}$ 附近, 两个峰相距 $57 \mathrm{~cm}^{-1}$, 在 $\mathrm{Nd}$ $(\mathrm{PMTFP})_{3}\left(\mathrm{Ph}_{3} \mathrm{PO}\right)_{2}$ 中, 前者红移到 $1637 \mathrm{~cm}^{-1}$ 处; 后者紫移到 $1665 \mathrm{~cm}^{-1}$. 两者相距 $28 \mathrm{~cm}^{-1}$. 在 $\mathrm{Nd}(\mathrm{PMTFP})_{3}(\mathrm{PMP}) \cdot \mathrm{H}_{2} \mathrm{O}$ 中, 两者进一步靠近, 只相差 $10 \mathrm{~cm}^{-1}$, 而在 $\mathrm{Nd}(\mathrm{PMTFP})_{3} \cdot$ $2 \mathrm{H}_{2} \mathrm{O}$ 中两者几乎合二而一, 但仍能在 $1635 \mathrm{~cm}^{-1}$ 的盽位左侧找到一个不明显的肩峰, 我们认 为发生红移的那个蜂相当于吡唑啉酮 5 位上的基, 在配位后有所削弱. 而发生紫移的那个 位为 4 位酰基上的崖基,在配位后, 由于互变异构形式的不复存在, 使单双键的平均化得到加 强. 这一变化可形象地用下式表示,同时为结构数据所证实.

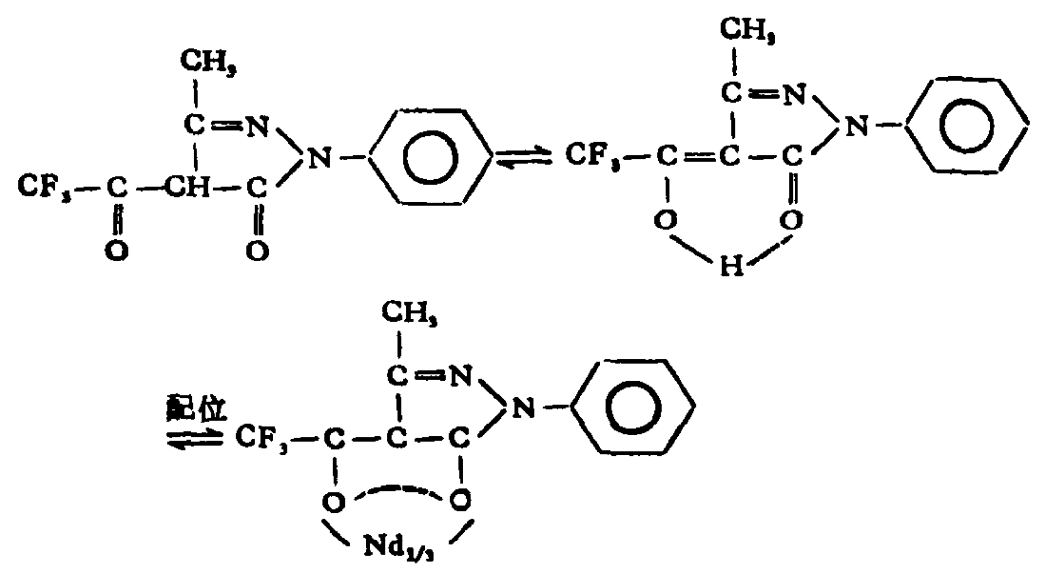

\section{二、 Nd(PMTFP $) \cdot($ Ph PO )，的晶体结构}

1. 四体枯构的测定与传正取大小为 $0.3 \times 0.3 \times 0.4 \mathrm{~mm}$ 的标题化合物单晶置于 EnrafNonius CAD4 四圆衍射仪上收集衍射数据. 辐射为经石䁷单色化的 Cu Ka $\mathrm{K}_{\mathrm{a}}$ 线共收集独立 行射点 7132 个, 其中 4935 个 $I>3 \sigma\left(I_{0}\right)$ 的衍射点用于结构测定和修正. 收集过程中三个 监测点强度无显著变化, 强度数据经 $L P$ 因子和经验吸收因子校正.

测定结果表明, 晶体属三斜晶系, 空间群为 $\mathrm{PI}$, 晶胞参数为 $a-12.880(4), b-13.649$. (7), $c-21.670(5) \AA, \alpha-93.14(3), \beta=91.63(2), \gamma-115.99(4)^{\circ}, V-3413.6 \AA^{3}$, $Z=2, D_{c}-1.48 \mathrm{gcm}^{-3}$.

分子式为

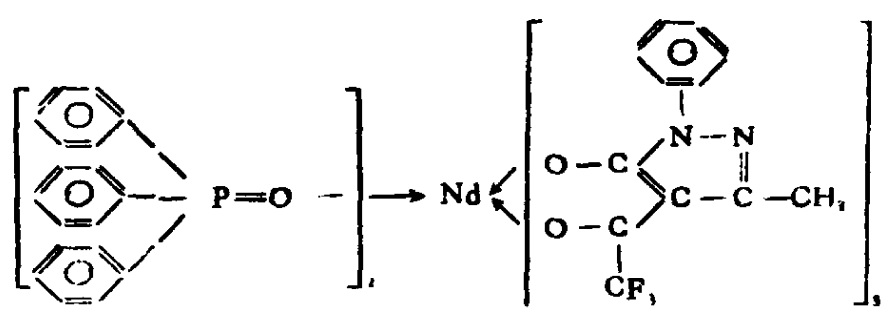

晶体结构由 Paffevson-Founier 法解出. 结构经全矩阵最小二乘法修正至收敛时 $R=$ 
表 1 原子分数坐标、等价各向同性热参数及标准偏差

\begin{tabular}{|c|c|c|c|c|}
\hline 原子 & $x$ & $\mathbf{Y}$ & $\mathbf{Z}$ & $B($ eq. $)$ \\
\hline ND(1) & $0.30345(5)$ & $0.45342(5)$ & $0.22924(3)$ & $3.36(1)$ \\
\hline$P(1)$ & $0.5550(2)$ & $0.4137(2)$ & $0.1645(1)$ & $3.89(7)$ \\
\hline$P(2)$ & $0.2738(3)$ & $0.2318(3)$ & $0.3374(2)$ & $4.62(7)$ \\
\hline$O(1)$ & $0.4527(6)$ & $0.4261(6)$ & $0.1824(4)$ & $4.3(2)$ \\
\hline$O(2)$ & $0.2974(7)$ & $0.3098(6)$ & $0.2897(4)$ & $4.7(2)$ \\
\hline$O(11)$ & $0.0982(7)$ & $0.3501(6)$ & $0.2537(4)$ & $4.7(2)$ \\
\hline$O(12)$ & $0.2407(7)$ & $0.5653(7)$ & $0.2938(4)$ & $5.1(2)$ \\
\hline$O(21)$ & $0.4490(7)$ & $0.5521(6)$ & $0.3155(4)$ & $4.8(2)$ \\
\hline$O(22)$ & $0.4321(7)$ & $0.6279(7)$ & $0.2030(4)$ & $4.5(2)$ \\
\hline$O(31)$ & $0.1977(6)$ & $0.2952(6)$ & $0.1515(4)$ & $4.4(2)$ \\
\hline$O(32)$ & $0.2072(6)$ & $0.4987(7)$ & $0.1526(4)$ & $4.8(2)$ \\
\hline$N(11)$ & $0.1604(8)$ & $0.6162(8)$ & $0.3756(5)$ & $4.2(2)$ \\
\hline$N(12)$ & $0.0631(8)$ & $0.5670(8)$ & $0.4108(5)$ & $4.5(2)$ \\
\hline$N(21)$ & $0.5721(9)$ & $0.8047(8)$ & $0.2032(5)$ & $5.0(2)$ \\
\hline$N(22)$ & $0.658(1)$ & $0.889(1)$ & $0.2425(7)$ & $7.0(3)$ \\
\hline $\mathbf{N}(31)$ & $0.0354(9)$ & $0.485+(8)$ & $0.1077(5)$ & $4.9(2)$ \\
\hline$N(32)$ & $-0.069(1)$ & $0.401(1)$ & $0.0802(6)$ & $6.0(3)$ \\
\hline$C(11)$ & $0.0339(9)$ & $0.3609(9)$ & $0.2940(6)$ & $3.8(2)$ \\
\hline$C(12)$ & $0.058(1)$ & $0.4132(9)$ & $0.3350(6)$ & $4.2(2)$ \\
\hline$C(13)$ & $0.160(1)$ & $0.5443(9)$ & $0.3304(6)$ & $4.3(2)$ \\
\hline$C(14)$ & $0.005(1)$ & $0.469(1)$ & $0.3877(6)$ & $4.5(2)$ \\
\hline$C(15)$ & $-0.100(1)$ & $0.398(1)$ & $0.4173(7)$ & $5.7(3)$ \\
\hline$C(16)$ & $-0.087(1)$ & $0.263(1)$ & $0.2875(6)$ & $4.6(2)$ \\
\hline$C(17)$ & $0.239(1)$ & $0.7262(9)$ & $0.3915(6)$ & $4.2(2)$ \\
\hline$C(18)$ & $0.327(2)$ & $0.779(1)$ & $0.354(1)$ & $7.5(4)$ \\
\hline$C(19)$ & $0.403(2)$ & $0.890(2)$ & $0.373(1)$ & $8.2(4)$ \\
\hline$C(110)$ & $0.388(2)$ & $0.938(1)$ & $0.4254(9)$ & $7.2(4)$ \\
\hline$C(111)$ & $0.30 .3(1)$ & $0.887(1)$ & $0.4603(9)$ & $7.1(4)$ \\
\hline$C(112)$ & $0.220(1)$ & $0.774(1)$ & $0.4420(8)$ & $6.1(3)$ \\
\hline$C(21)$ & $0.523(1)$ & $0.644(1)$ & $0.3287(6)$ & $4.8(3)$ \\
\hline$C(22)$ & $0.561(1)$ & $0.7262(9)$ & $0.2919(6)$ & $4.2(2)$ \\
\hline$c(23)$ & $0.5139(9)$ & $0.7129(8)$ & $0.2300(5)$ & $3.5(2)$ \\
\hline$C(24)$ & $0.649(2)$ & $0.839(1)$ & $0.2938(9)$ & $7.2(4)$ \\
\hline$C(25)$ & $0.732(2)$ & $0.915(2)$ & $0.348(1)$ & $9.6(6)$ \\
\hline$C(26)$ & $0.576(2)$ & $0.672(2)$ & $0.397(1)$ & $7.6(4)$ \\
\hline$C(27)$ & $0.55+(1)$ & $0.828(1)$ & $0.1421(8)$ & $5.9(3)$ \\
\hline$C(28)$ & $0.447(1)$ & $0.782(1)$ & $0.1127(9)$ & $7.1(4)$ \\
\hline$C(29)$ & $0.433(2)$ & $0.807(2)$ & $0.051(1)$ & $9.2(5)$ \\
\hline$C(210)$ & $0.531(2)$ & $0.876(2)$ & $0.023(1)$ & $10.1(6)$ \\
\hline$C(211)$ & $0.631(2)$ & $0.919(2)$ & $0.049(1)$ & $9.8(6)$ \\
\hline$C(212)$ & $0.652(2)$ & $0.895(2)$ & $0.112(1)$ & $8.1(4)$ \\
\hline$C(31)$ & $0.106(1)$ & $0.2673(9)$ & $0.1214(6)$ & $4.3(2)$ \\
\hline$c(32)$ & $0.051(1)$ & $0.330(1)$ & $0.1082(7)$ & $4.9(3)$ \\
\hline$C(33)$ & $0.1106(9)$ & $0.4451(9)$ & $0.1270(6)$ & $3.8(2)$ \\
\hline$C(34)$ & $-0.062(1)$ & $0.314(1)$ & $0.0807(8)$ & $6.0(3)$ \\
\hline$C(35)$ & $-0.160(2)$ & $\cdot 0.213(1)$ & $0.054(1)$ & $7.6(4)$ \\
\hline$c(36)$ & $0.053(1)$ & $0.151(1)$ & $0.0957(8)$ & $6.4(3)$ \\
\hline$c(37)$ & $0.046(1)$ & $0.590(1)$ & $0.1129(7)$ & $5.4(3)$ \\
\hline$C(38)$ & $-0.028(1)$ & $0.620(1)$ & $0.0770(9)$ & $7.1(4)$ \\
\hline$C(39)$ & $-0.013(2)$ & $0.729(2)$ & $0.087(1)$ & $9.0(5)$ \\
\hline
\end{tabular}


续表 !

\begin{tabular}{|c|c|c|c|c|}
\hline 辰于 & $x$ & $Y$ & $z$ & $B$ (eq.) \\
\hline$C(310)$ & $0.065(2)$ & $0.806(2)$ & $0.125(1)$ & $8.4(5)$ \\
\hline$c(311)$ & $0.138(2)$ & $0.774(2)$ & $0.160(1)$ & $8.7(5)$ \\
\hline$c(312)$ & $0.128(1)$ & $0.668(1)$ & $0.1553(8)$ & $6.3(3)$ \\
\hline$C(41)$ & $0.603(1)$ & $0.4733(9)$ & $0.0951(6)$ & $4.1(2)$ \\
\hline$C(42)$ & $0.597(1)$ & $0.570(1)$ & $0.0858(7)$ & $5.4(3)$ \\
\hline$C(43)$ & $0.633(1)$ & $0.622(1)$ & $0.0304(8)$ & $6.2(3)$ \\
\hline$C(44)$ & $0.677(1)$ & $0.578(1)$ & $-0.0135(8)$ & $6.6(3)$ \\
\hline$C(45)$ & $0.683(1)$ & $0.483(1)$ & $-0.0045(8)$ & $6.5(3)$ \\
\hline$C(46)$ & $0.648(1)$ & $0.430(1)$ & $0.0497(7)$ & $5.5(3)$ \\
\hline$C(51)$ & $0.6741(9)$ & $0.4799(9)$ & $0.2215(5)$ & $3.7(2)$ \\
\hline$c(52)$ & $0.783(1)$ & $0.533(1)$ & $0.2063(8)$ & $6.2(3)$ \\
\hline$c(53)$ & $0.872(2)$ & $0.585(1)$ & $0.2536(9)$ & $7.4(4)$ \\
\hline$c(54)$ & $0.845(2)$ & $0.575(1)$ & $0.3144(9)$ & $7.2(4)$ \\
\hline$c(55)$ & $0.736(1)$ & $0.521(1)$ & $0.3298(6)$ & $6.3(3)$ \\
\hline$c(56)$ & $0.648(1)$ & $0.469(1)$ & $0.2841(8)$ & $6.1(3)$ \\
\hline$C(61)$ & $0.523(1)$ & $0.273(1)$ & $0.1575(6)$ & $4.5(2)$ \\
\hline$C(62)$ & $0.607(1)$ & $0.236(1)$ & $0.1648(8)$ & $6.0(3)$ \\
\hline$C(63)$ & $0.577(2)$ & $0.124(1)$ & $0.1621(9)$ & $7.4(4)$ \\
\hline$C(64)$ & $0.463(2)$ & $0.052(2)$ & $0.151(1)$ & $8.3(5)$ \\
\hline$C(65)$ & $0.378(2)$ & $0.087(2)$ & $0.144(1)$ & $8.0(4)$ \\
\hline$C(66)$ & $0.408(1)$ & $0.199(1)$ & $0.1462(7)$ & $5.3(3)$ \\
\hline$C(71)$ & $0.217(1)$ & $0.270(1)$ & $0.4022(6)$ & $4.6(2)$ \\
\hline$C(72)$ & $0.261(1)$ & $0.383(1)$ & $0.4197(7)$ & $5.4(3)$ \\
\hline$c(73)$ & $0.212(2)$ & $0.418(2)$ & $0.469(1)$ & $8.0(4)$ \\
\hline$C(74)$ & $0.871(2)$ & $0.657(2)$ & $0.498(1)$ & $8.2(4)$ \\
\hline$C(75)$ & $0.917(2)$ & $0.766(2)$ & $0.516(1)$ & $9.0(5)$ \\
\hline$c(76)$ & $0.131(1)$ & $0.197(1)$ & $0.4338(8)$ & $6.5(3)$ \\
\hline$C(81)$ & $0.172(1)$ & $0.100(1)$ & $0.3116(7)$ & $4.9(3)$ \\
\hline$C(82)$ & $0.171(1)$ & $0.005(1)$ & $0.3369(9)$ & $7.0(4)$ \\
\hline$c(83)$ & $0.080(2)$ & $0.902(2)$ & $0.319(1)$ & $8.7(5)$ \\
\hline$C(84)$ & $-0.004(2)$ & $0.893(2)$ & $0.276(1)$ & $9.4(5)$ \\
\hline$C(85)$ & $-0.006(2)$ & $-0.015(2)$ & $0.252(1)$ & $8.0(4)$ \\
\hline$C(86)$ & $0.084(1)$ & $0.088(1)$ & $0.2679(7)$ & $5.7(3)$ \\
\hline$C(y 1)$ & $0.402(1)$ & $0.219(1)$ & $0.3631(7)$ & $4.9(3)$ \\
\hline$C(92)$ & $0.453(1)$ & $0.183(1)$ & $0.3197(8)$ & $6.0(3)$ \\
\hline$C(93)$ & $0.555(2)$ & $0.175(1)$ & $0.3354(9)$ & $7.3(4)$ \\
\hline$C(94)$ & $0.601(2)$ & $0.207(1)$ & $0.395(1)$ & $7.5(4)$ \\
\hline$C(95)$ & $0.556(2)$ & $0.249(2)$ & $0.438(1)$ & $8.0(4)$ \\
\hline$C(96)$ & $0.452(1)$ & $0.255(1)$ & $0.4225(9)$ & $6.8(4)$ \\
\hline$F(11)$ & $-0.1077(9)$ & $0.2002(9)$ & $0.3350(5)$ & $8.3(2)$ \\
\hline$F(12)$ & $-0.1052(8)$ & $0.1956(8)$ & $0.2382(5)$ & $7.7(2)$ \\
\hline$F(13)$ & $-0.1728(9)$ & $0.2920(8)$ & $0.2876(5)$ & $7.9(2)$ \\
\hline$F(21)$ & $0.691(1)$ & $0.707(1)$ & $0.4009(7)$ & $10 .+(3)$ \\
\hline$F(22)$ & $0.5+1(1)$ & $0.581(1)$ & $0.4266(8)$ & $11.8(4)$ \\
\hline$F(23)$ & $0.557(1)$ & $0.746(1)$ & $0.4273(7)$ & $10 .+(3)$ \\
\hline $\mathbf{F}(31)$ & $0.121(1)$ & $0.1015(9)$ & $0.1032(6)$ & $8.8(3)$ \\
\hline$F(32)$ & $-0.047(1)$ & $0.086(1)$ & $0.1194(6)$ & $9.2(3)$ \\
\hline$F(33)$ & $0.027(1)$ & $0.134(1)$ & $0.0330(6)$ & $9.1(3)$ \\
\hline
\end{tabular}


ก.089, $R_{w}=0.091$, 最后一次修正中采用了 Dunitz-Seiler 修正的单位权重方式 . 最终羑 值电子密度上最高峰小于 $1 e / \AA^{3}$, 没有企图确定氢原子位置。

表 1 列出 98 个非氢原子分数坐标及其等价各向同性热参数，图 1 为分子立体结 抅透炠 图.

结抱分析表明,晶体由孤立分子组成，每 一不对称单位中含有一个分子，分子式符合 $\mathrm{Nd}(\mathrm{PMTFP})_{3}\left(\mathrm{Ph}_{3} \mathrm{PO}\right)_{2}$ 。钕原子处于由八个 拿原子组成的四方反棱柱体中心, 其中六个 氧原子来自三个双齿配体 PMTFP 基, 其 余两个来自两个单齿配体 $\mathrm{Ph}_{3} \mathrm{PO}$. 原子组 $O(1) 、 O(22) 、 O(31), O(32)$ 和 $O(2) 、 O$ (11)、O(12)，O(21) 组成正方形. 以上两原 子组各自的最小二乘平面间夹角为 $1.4^{\circ}$, 即 两平面基本平行。

三个 $\beta$-二酮 PMTFP 基均以双齿形式 与中心离子 $\mathrm{Nd}$ 配位. 那些靠近 $\mathrm{CF}_{3}$-基团 的氧原子 $O(11) O(21) O(31)$ 所形成的 $\mathrm{Nd}-\mathrm{O}$ 键的键长分别为 $2.480,2.477 .2 .499$

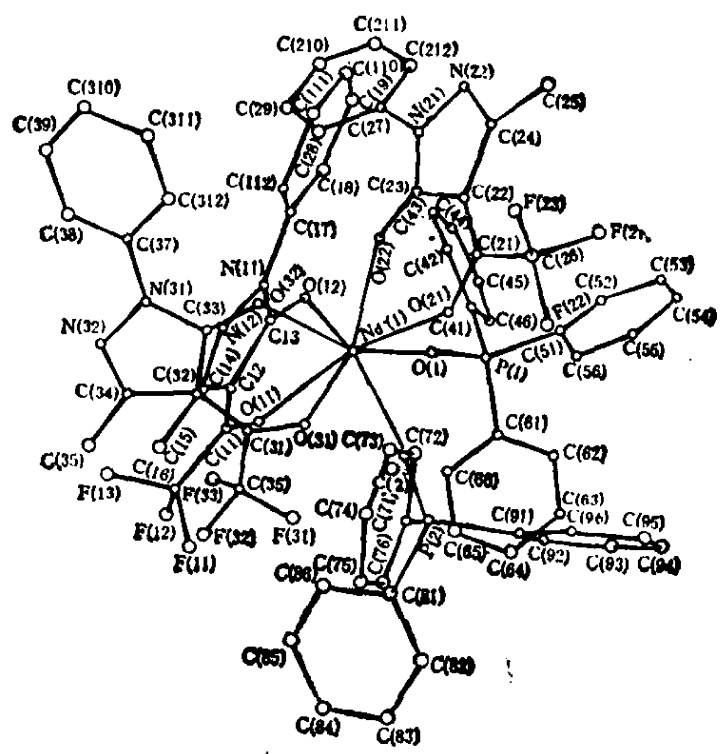

图 $1 \mathrm{Nd}(\mathrm{PMTFP})_{3}(\mathrm{Ph}, \mathrm{PO})_{2}$ 分子结构透枧图 $\AA$ 比那些靠近苯环的氧原子 $\mathrm{O}(12) 、 \mathrm{O}(22) 、 \mathrm{O}(32)$ 所形成的 $\mathrm{Nd}-\mathrm{O}$ 键分别为 $2.420 、 2.342$ 、 $2.312 \AA$ 要长. $\beta$ 二酮上的 $\mathrm{C}=\mathrm{O}$ 键也可以分为两组, 靠近 $\mathrm{CF}_{3}^{-}$基团一边的 $\mathrm{C}=\mathrm{O}$ 键长 分别为 $1.267(4) 、 1.206(4) 、 1.223(4) \AA$ 平均值为 $1.235(4) \AA$ 而靠近苯环一边的 $\mathrm{C}=\mathrm{O}$ 键长 分别为 $1.262(5) 、 1.272(4) 、 1.227$ (4) 平均值为 $1.254(5) \AA$, 前者比后者为短, 造成以上现象的 原因可能是由于强电负性的 $\mathrm{CF}^{-}$基团吸电子作用, 使靠近它的氧原子上的电子云转移的缘 故.

\section{考文 却}

[1] Jensen, B. S., Acta Chem. Scand., 1959, 8: 13.

[2]萗启家、毛家悛、金忠、陆志仁,化学分离法,原子能出版社, 1984 .

[3] Huang Chun-hui and Freiser, H., Solvent Extraction ond lon Exchonge, 4(1986), 4: $41-59$.

[4] 顾瑟东、末源, 高等学校化学学报, 1981,2: 139.

[5] Dunitz, J. D.and Seiler, P., Acta Cryst., B29 (1973), 589. 